\title{
Analyzing Emotion Patterns based on Cutaneous Temperature
}

\author{
G. A. do Nascimento \\ PUC Goiás \\ Goiânia, Brazil
}

\author{
E. L. da Costa \\ PUC Goiás \\ Goiânia, Brazil
}

\author{
S. G. S. Alcalá \\ UFG \\ Aparecida de Goiânia, \\ Brazil
}

\author{
T. M. G. de A.Barbosa \\ PUC Goiás \\ Goiânia, Brazil
}

\begin{abstract}
Physical and psychological diseases are problems that may be difficult to detect before they reach serious stages. However, the human body can manifest minute signals that can be identified and interpreted as warnings about a person's health. Consequently, preventative actions can be taken. Even though it has been a challenge, due to different signals patterns between subjects, these signals can be collected and analyzed by devices which can offer real-time feedback for the user. This article presents a study based on cutaneous temperatures collected from undergraduate students when stimulated to different kinds of emotions. The goal is to correlate the kinds of emotions with specific temperature patterns observed. The results are satisfactory once it was possible to observe a pattern in temperature variation during situations of stress and relaxation.
\end{abstract}

\section{General Terms}

Affective Computing.

\section{Keywords}

Affective computing, stress, cutaneous temperature.

\section{INTRODUCTION}

Affective computing is the human-computer interaction in which a device is able to detect, to process or to influence emotive situations. It combines the fields of computer science, psychology and cognitive science in order to interpret emotive states and to give appropriate feedbacks to these patterns [1].

The acquisition and online processing of physiology signals can be of great importance in detection and prevention of diseases [2], customers' impact evaluation when watching advertisements, or even to the development of adaptive games [3]. Accuracy and response time are among the main factors that contribute to the affective computing use in these cases. For example, within medical applications, the early detection of a disease might be crucially important to determine either a patient must go through a preventive procedure or a cure treatment. In this kind of application, the patient can carry a device that automatically measure physiological levels and give to him an instantaneous response, in order to replace medical appointments by a full time monitoring.

The use of cutaneous temperature as a way to obtain physiological feedbacks to the sympathetic nervous system (SNS) offers a valid indicator to the emotional state detection [4]. Studies prove that there exist a relation between stimulus to the SNS and the temperature variation at the fingers [5]. The method is also a low cost alternative and less invasive, once it is necessary only the skin contact to capture the temperature signals.
In this project, analysis based on cutaneous temperature datasets collected from human beings will be made. The process was executed in humans when subjected to stimulus that induced them to emotional states such as relaxation, stress and scare situations.

\section{MATERIALS AND METHODS}

One of the requirements of the temperature collect system is the capacity to be attached to the user's daily life as a noninvasive device and to not represent any sort of discomfort and insecurity. Furthermore, the system must to be fast responding, once that small changes in temperature levels must be detected. Considering these aspects, the acquisition method chosen is the one proposed by Begun [2], where the cutaneous temperature at the fingers is used as an indicator.

Differently from other acquisition methods that capture physiological signals, such as galvanic skin resistance and electrocardiogram, the system proposed by Begun does not require the user to be part of it, avoiding discomforts generated by these sort of methods, for example stimulus by an electric current or pressure on skin. The sensor also does not require that conduct materials get in touch with the user's skin, excluding any use of a circuit protection.

The proposed circuit to this experiment is presented in Figure 1. The circuit is able to read temperatures in the range between $10^{\circ} \mathrm{C}$ and $45^{\circ} \mathrm{C}$. These values were chosen based on the temperature variation of a human hand when subject to stress situations [5] in a temperature environment set in approximately $23^{\circ} \mathrm{C}$

According to some factors, such as accuracy requirements, adaptability and comfort, the temperature sensor used in the circuit was the thermistor B57861S0103F45 from EPCOS. This thermistor has an internal resistance of $10 \mathrm{k} \Omega$ at $25^{\circ} \mathrm{C}$ and flexible wires, which allows an easy adaptability to any equipment without causing discomfort to the user.

The current source REF200 was used to effectuate the reading of the sensor. An approaching using a voltage divider would overheat the resistor utilized in the circuit, because the thermistor's resistance has a large variation for the interested interval (from $4,37 \mathrm{k} \Omega$ up to $19,9 \mathrm{k} \Omega$ ), also generating a large current variation. A filter was adopted to eliminate the frequencies originated from the external environment. 


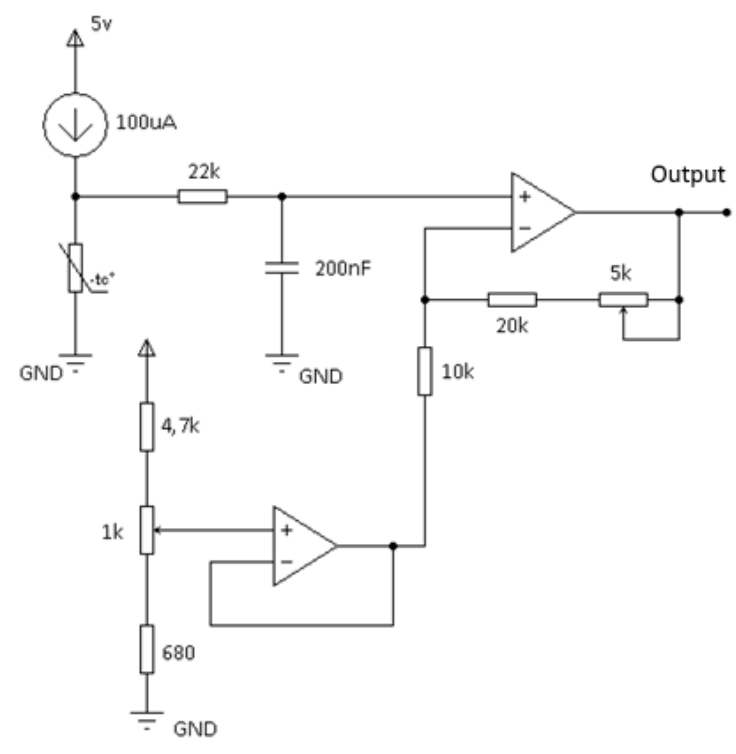

Figure 1: Acquisition temperature circuit.

Since the sensor's potential difference for the interested interval ranges from $0.437 \mathrm{~V}$ up to $1.99 \mathrm{~V}$ (corresponding to $1.553 \mathrm{~V}$ ) and the desired circuit output is in a band from $0 \mathrm{~V}$ to $5 \mathrm{~V}$, it was decided to include an operational amplifier subtractor. The microcontroller MSP430 was used to read the circuit. It has an ADC (Analog-to-Digital Converter) of 12 bits, offering $1.22 \mathrm{mV}$ of resolution, in other words, each $1.22 \mathrm{mV}$ variation at the circuit output represents 1 unit in the ADC conversion. Figure 2 shows the transfer function for the conversion from ADC values to temperature levels.

The collect circuit was attached to the Emopad [6], a system for acquisition of biological signals, such as galvanic resistance, heart frequency and temperature levels, which is attached to a video game control. The collects were taken during a presentation at Campus Party Brazil 2015. The average age of all invited users is 22 years and the experiment included both genders.

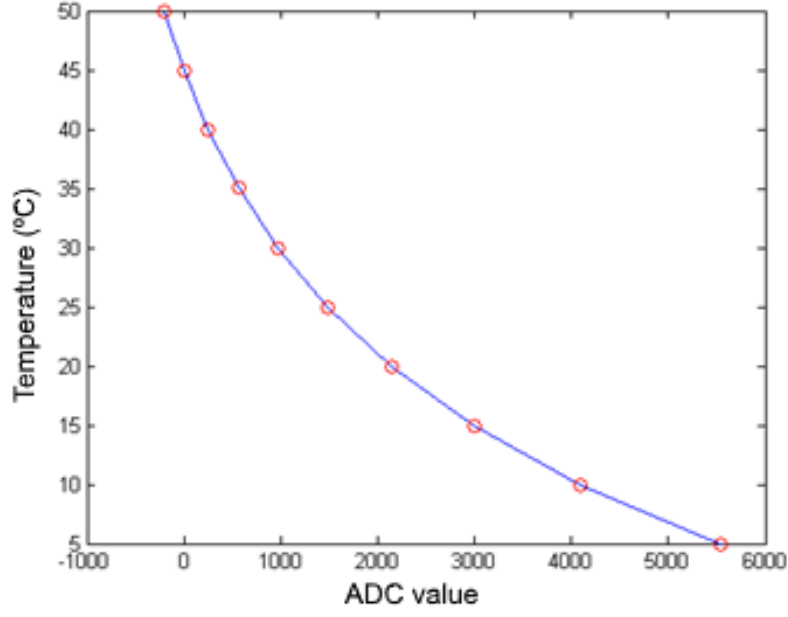

Figure 2: ADC-temperature transfer function for MSP430

At the first moment of the test, the users were oriented to stay in repose for 5 minutes, this part intends to balance the user body`s temperature to the environmental temperature. Next, the corporal temperature was measured using a clinical thermometer settled under the armpit to establish the average body's temperature at the moments previous to the experiment.

Then, the person it invited to sit down in a comfortable position, rightly holding the controller, in front of a monitor where a video of 16 minutes is exhibited. Figure 3 shows the video's timeline.

A second monitor was used by the system's administrator. This screen serves as a visual control of the equipment. A Matlab application was designed to receive, to store and to output the signal captured by the microcontroller. Figure 4 shows the Matlab application.

The exhibited video aims to stimulate some reactions to the users, such as relaxation, scare and stress. The user is asked to stay in the same position for the whole time, without moving drastically. Headphones are also used for the experiment. Figure 5 shows the moment of the collect.

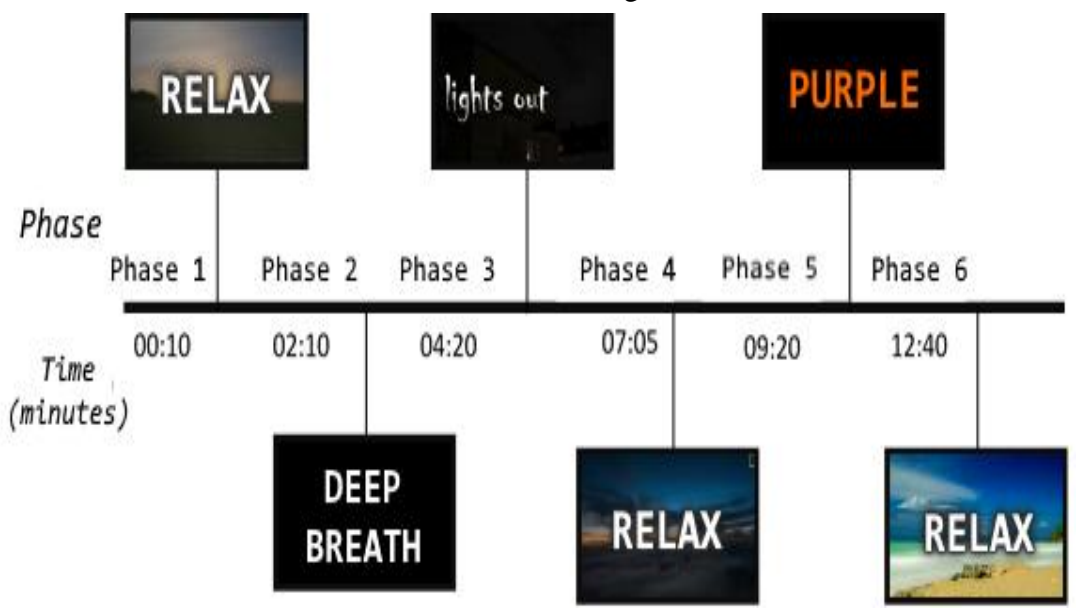

Figure 3: Timeline of the video 


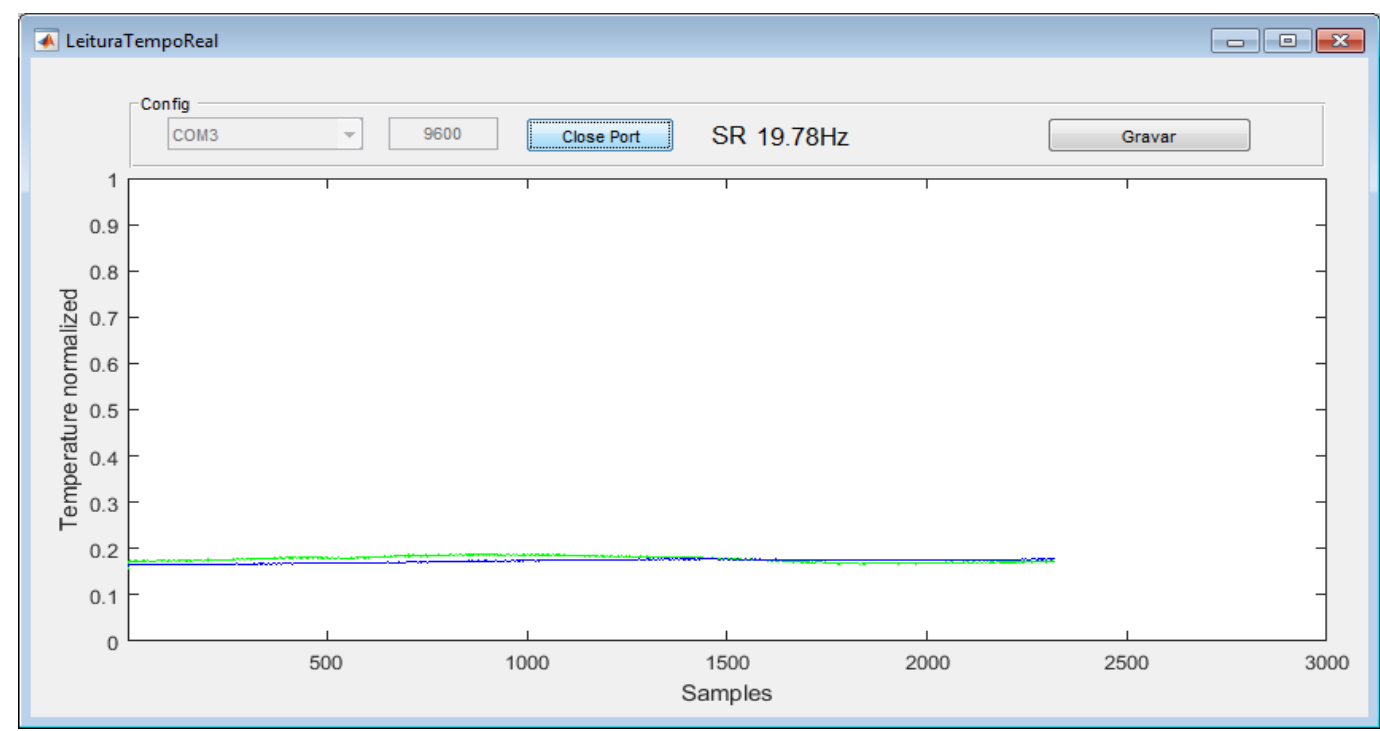

Figure 4: Interface designed in Matlab to output the signal

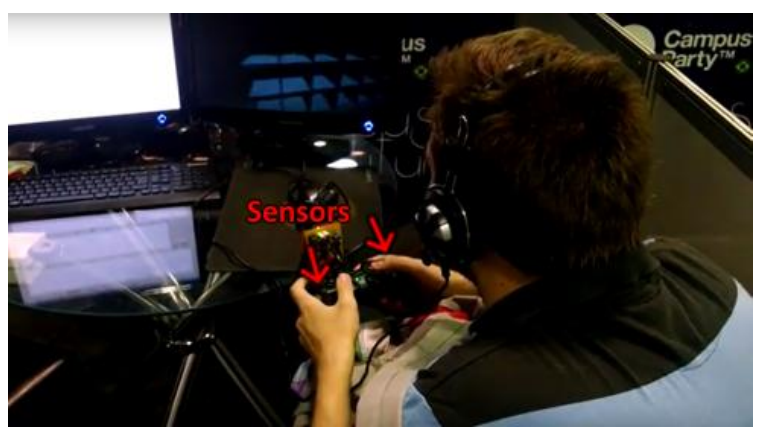

Figure 5: Experiment time. The red arrows indicate the thermistor's position

In phases 1, 4 and 6 are exhibited landscapes with a classic background music. These steps are strategically inserted in the beginning of the video, right after stressful moments. They are intended to induce relaxation to the user and to normalize its body's temperature. In that way it is possible to give the user a short time between two opposite phases to recuperate its normal emotional state.

Phases 2, 3 and 5 ate intended to observe the cutaneous temperature variation. They are designed to obtain relaxation, scare and mental stress respectively. The Stroop Colour Test, proposed by John Stroop and described in [7], was used to stimulate stress.

For the purpose of adding a second reaction examiner, the users were recorded during the moment of collect. Table 1 relates the video phases and the expected reactions.

At the end of the test the users were asked to comment each phase of the video, so that was possible to confirm if the expected reaction for each phase was reached indeed.
Table 1: Description of the video's phases and expected reactions

\begin{tabular}{|c|c|c|}
\hline Phase & Stimulus & Expected Reaction \\
\hline Phase 1 & Soothing scenes & $\begin{array}{l}\text { Normalization of the user's } \\
\text { emotional state }\end{array}$ \\
\hline Phase 2 & Deep Breath & Relaxation \\
\hline Phase 3 & $\begin{array}{l}\text { Watch a thriller } \\
\text { scene }\end{array}$ & Scare \\
\hline Phase 4 & Soothing scenes & $\begin{array}{l}\text { Normalization of the user's } \\
\text { emotional state }\end{array}$ \\
\hline Phase 5 & $\begin{array}{l}\text { Stroop Colour Word } \\
\text { Test }\end{array}$ & Mental stress \\
\hline Phase 6 & Soothing scenes & $\begin{array}{l}\text { Normalization of the user's } \\
\text { emotional state }\end{array}$ \\
\hline
\end{tabular}

\section{RESULTS}

A total of 10 people were submitted to the experiment. Figure 6 shows the left hand's temperature variation for one of the participants.

Analyzing the figure, five moments of big variation can be observed over time. At phases 1, 4 and 6, where soothing scenes are exhibited, the user's cutaneous temperature shown a significant increment over time (less than $1^{\circ} \mathrm{C}$ ). Both phases 3 and 5, supposed to stimulate stress situations to the user, presented a temperature decrement of approximately $0.8^{\circ} \mathrm{C}$. Phase 2, intended to conduce the user to relaxation did not demonstrate a big variation as expected.

The same patterns were also observed when analyzing Figure 7 , which shows the right hand's temperature variation for the same user. However, were noticed smaller temperature levels in comparison to the right hand. Emotional arousal was previously studied by measuring the sympathetic nervous system activation and shown that it can differ across the two halves of the upper body [8]. 


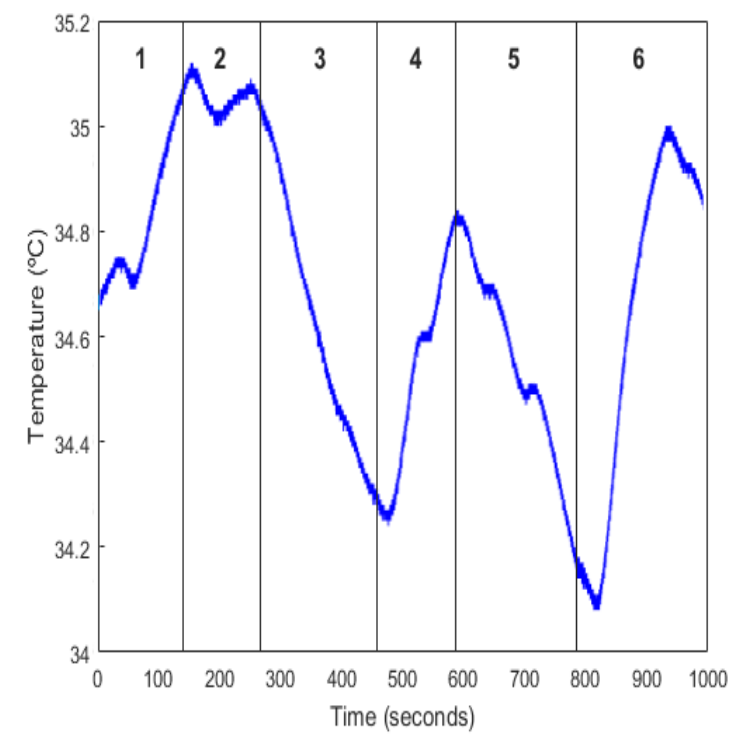

Figure 6: Left hand's temperature variation over the video for user 1

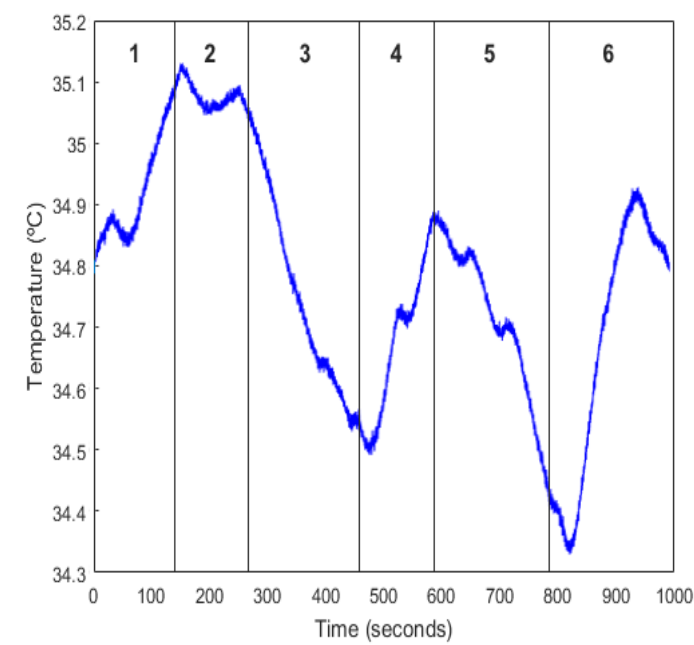

Figure 7: Right hand's temperature variation over the video for user 1

Figure 8 shows the temperature variation of the right hand for another user. Analyzing this graphic, it is possible to notice the same patterns presented by user 1 , but in a smaller scale. This user presented a maximum cutaneous temperature variation of $0.35^{\circ} \mathrm{C}$. The biggest range observed was in phase 5 , showing that the Stroop Colour Test presented the expected results. Phases 1, 4 and 6 show the temperature's level incrementing, confirming the efficiency of the soothing scenes chosen for the experiment.

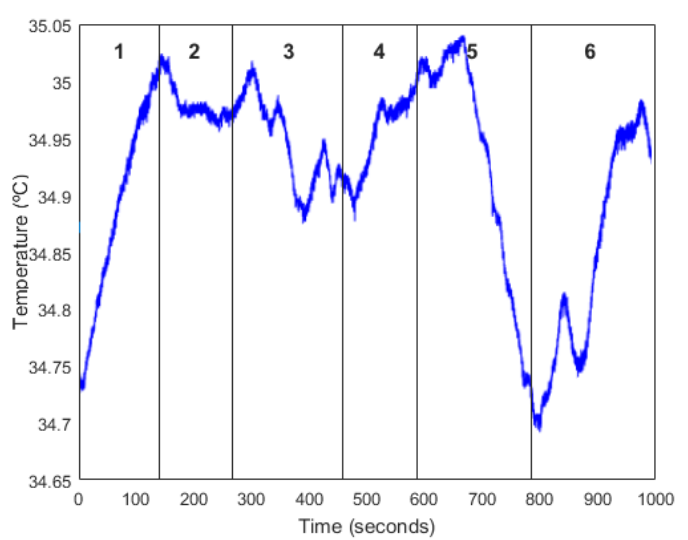

Figure 8: Right hand's temperature variation over the video for user 2

Figure 8 show the right hand's temperature variation for user 3 . It is easily observed the temperature variation at phases 3 , 4, 5 and 6. However, phases 1 and 2 did not presented results as expected. When questioned about the experiment, user 3 reported to not be able to relax during the initial phases as the video asked to.

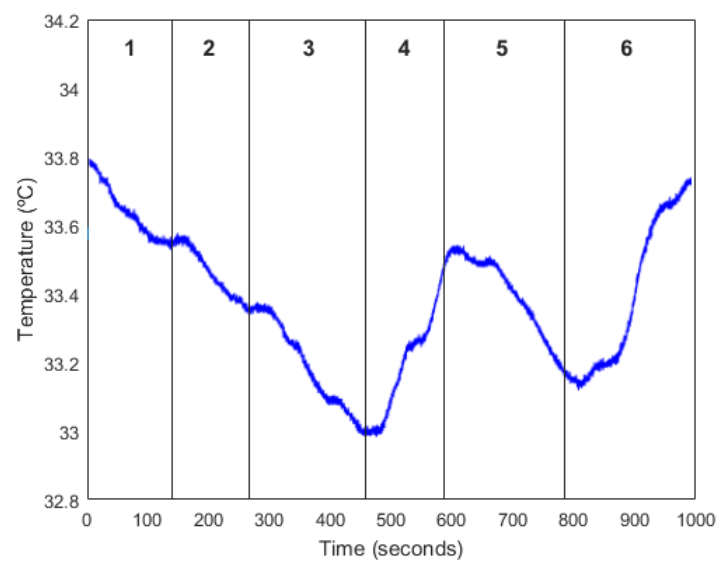

Figure 8: Right hand's temperature variation over the video for user 3

Some collects did not present visible temperature variation. There were cases where the temperature declined over the 16 minutes video and other cases where was observed the temperature raising up. Both situations represented small changes between the video's phases.

\section{DISCUSSIONS}

At the end of the experiment, all the users were asked to comment about the feelings they had during the main video's phases. Most phases had the expected stimuli.

Analyzing Table 1 it is possible to observe that there was an agreement between the expected patterns and the results obtained when the users followed the video's instructions. Phases 3 and 5, intended to stimulate scare and stress, as introduced by Kister et al. [5], presented a decay of the temperature in $80 \%$ of the users.

Steps 1, 4 and 6, destined to balance the user's cutaneous temperature (to the normal temperature), show an increment of the temperature in $90 \%$ of the users. However, it was not a 
complete recuperation. This behavior is easily observed during phase 4 , where the highest reached temperature was approximately $34.8^{\circ} \mathrm{C}$ for user 1 , resulting in a difference of about $0.3^{\circ} \mathrm{C}$ in comparison to the maximum temperature observed during the first relaxing scene.

Phase 2 (intended to induce the user to relaxation) did not presented the expected result. It was expected the cutaneous temperature to rise, but the variation acquired had a small change or no change in the collects.

\section{CONCLUSION}

The experiment presented satisfactory results. It was possible to prove the relation between the cutaneous temperature variation and the user's emotional state.

The Stroop Colour Word Test had shown to be a great mental stress stimulator as the scare scene. These two phases will be maintained for the next experiments, without any alterations.

The soothing scenes also presented good results. However, it was noticed that the dedicated time for these scenes (about 2 minutes) was not enough to induce the user to its normal emotional state. The same scenes will be exhibited in future experiments, but with longer duration.

Studies will be made to identify methods to induce the user to a better relaxation state. It was noticed that the act of taking a deep breath was not a good indicator for the purpose of this experiment.

After correcting the identified improvements points in this experiment, artificial intelligence techniques will be used to develop a software that automatically detects the user's current emotional state. These changes will allow to build a circuit that fits in the user's daily life, so that will be possible to monitor its physical and emotional state in real time.

\section{REFERENCES}

[1] Picard RW. Affective Computing. Cambridge, MA: Mit Press; 1997. 304 p.

[2] Begum S. A case-based reasoning system for the diagnosis of individual sensitivity to stress in psychophysiology. 2009. $64 \mathrm{f}$. PhD Thesis - Computer Science, School of Innovation, Design and Engineering, Mälardalen University, Västerås, Suécia, 2009.

[3] Hudlicka E. Affective computing for game design. In: Proceedings of the 4th International North American Conference on Intelligent Games and Simulation (GAMEON-NA); 2008 Ago 13-15; McGill University, Montreal, Canada. 2008. p. 5-12.

[4] Kistler A, et al. Acute sympathetic responses elicited by acupuncture are pain-related and non-specific. Karger. 1996; 3(6):269-278.

[5] Kister A, Mariauzouls C, von Berlepsch K. Fingertip temperature as an indicator for sympathetic responses. International Journal of Psychophysiology. 1998; 29(1):35-41.

[6] Bacchini PHF, Ferreira JO, Barbosa TMGA. Emopad: An Affective Gamepad. International Journal of Computer Applications. 2014 Sep; 102(15):5-12.

[7] Bilge P, Nezih D, Dicle B. Computer adapted stroop colour-word conflict test as a laboratory stress model. Erciyes Medical Journal. 2005; 27(2):58-63.

[8] Picard, R. W., Fedor, S., \& Ayzenberg Y. Multiple Arousal Theory and Daily-Life Electrodermal Activity Asymmetry. Emotion Review, March 2015. 